\title{
Retroviral DNA integration and the DNA damage response
}

\author{
AM Skalka ${ }^{*, 1}$ and RA Katz ${ }^{1}$ \\ ${ }^{1}$ Fox Chase Cancer Center, Institute for Cancer Research, 333 Cottman \\ Avenue, Philadelphia, PA 19111-2497, USA \\ * Corresponding author: AM Skalka, Fox Chase Cancer Center, Institute for \\ Cancer Research, 333 Cottman Avenue, Philadelphia, PA 19111-2497, USA. \\ Tel: +215-728-2490; Fax: +215-728-2778; E-mail: am_skalka@fccc.edu
}

Received 02.11.04; revised 03.12.04; accepted 06.12.04; published online 11.3.05 Edited by $\mathrm{G}$ Kroemer

\begin{abstract}
Retroviral DNA integration creates a discontinuity in the host cell chromatin and repair of this damage is required to complete the integration process. As integration and repair are essential for both viral replication and cell survival, it is possible that specific interactions with the host DNA repair systems might provide new cellular targets for human immunodeficiency virus therapy. Various genetic, pharmacological, and biochemical studies have provided strong evidence that postintegration DNA repair depends on components of the nonhomologous end-joining (NHEJ) pathway (DNA-PK (DNA-dependent protein kinase), Ku, Xrcc4, DNA ligase IV) and DNA damage-sensing pathways (Atr (Atm and Rad related), $\gamma$-H2AX). Furthermore, deficiencies in NHEJ components result in susceptibility to apoptotic cell death following retroviral infection. Here, we review these findings and discuss other ways that retroviral DNA intermediates may interact with the host DNA damage signaling and repair pathways.

Cell Death and Differentiation (2005) 12, 971-978.

doi:10.1038/sj.cdd. 4401573

Published online 11 March 2005
\end{abstract}

Keywords: DNA repair pathways; apoptosis; integrase; NHEJ; DNA damage signaling

Abbreviations: DSB, double-strand break in DNA; NHEJ, nonhomologous end joining; HRR, homologous recombination repair; scid, severe combined immunodeficient; ASV, avian sarcoma virus; HIV-1, human immunodeficiency virus type 1; DNA-PK, DNA-dependent protein kinase; Atm, ataxia-telangiectasia mutated (kinase); Atr, Atm and rad-related protein (kinase)

\section{Introduction}

Viruses are obligate parasites that require host cell functions for their survival and propagation. Successful viruses have evolved mechanisms to exploit and/or counteract host defense systems, and modify or redirect normal cellular processes; many viral genomes are replicated and/or transcribed by host cell enzymes. In the last few years, it has been discovered that some viruses interact with the cellular DNA damage-sensing repair pathways. This diverse group includes adenoviruses, adeno-associated virus, and retroviruses. These interactions can either support or inhibit viral replication, but ultimately there is selection for survival of both the virus and the cell. Retroviral DNA integration causes damage to the host cell chromosome and unrepaired DNA damage can lead to apoptosis. This review focuses on the host cell response to retroviral DNA integration as a trigger for the cellular DNA damage response and how interruption of this interplay can lead to apoptotic cell death.

\section{Retroviral DNA Integration}

Retroviruses encode a recombinase protein, called integrase, which is required for the insertion of viral DNA into the chromatin of its host cell. ${ }^{1,2}$ This process is essential for retroviral replication and also ensures virus persistence for the life of the cell. The first two steps in the process are catalyzed by integrase, acting upon specific sequences at the ends of the viral DNA (Figure 1a). In the first step, denoted processing, two nucleotides are removed from the $3^{\prime}$-ends of the viral DNA, a reaction that can occur in the cytoplasm. In the second step, joining, the two newly created recessed $3^{\prime}$ ends are joined to staggered phosphorus atoms in the backbones of complementary strands of a host DNA, via a concerted cleavage-ligation reaction. As a consequence of integrase-mediated joining, the host cell DNA suffers a double-strand break (DSB), the ends of which are held together by single-strand links to viral DNA (Figure 1b, left).

Retroviral DNA integration produces a complex lesion in host DNA. Interruption of existing chromatin conformation and composition by the insertion of 3-10 kb of newly synthesized DNA is likely to be sensed as a major assault on the genomic integrity of the cell (Figure 2a). The DNA damage incurred includes two short single-stranded gaps in the host DNA flanking the integration site, and possibly single-strand interruptions at internal sites in the inserted viral DNA as well (Figures $1 \mathrm{~b}$ and $2 \mathrm{~b}$ ). ${ }^{3,4}$ Collision of a host cell replication fork with unrepaired gaps during S-phase (Figure 2c) could produce DSBs with free ends at the site of integration. In addition, unintegrated linear viral DNA molecules in the nucleus may resemble chromosomal fragments that can also be recognized by host DNA damage-sensing and repair pathways leading to self-ligation. ${ }^{5,6}$ It seems likely, however, that mechanisms to limit such self-ligation have evolved, as this reaction could compete with productive integration. In contrast, postintegration repair (Figure 1b) is essential for the maintenance of host DNA integrity as well as the stable, 
a Retroviral DNA Integration

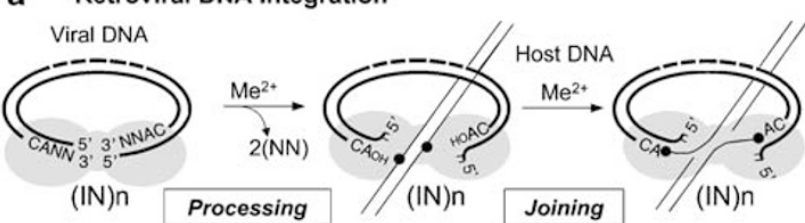

b

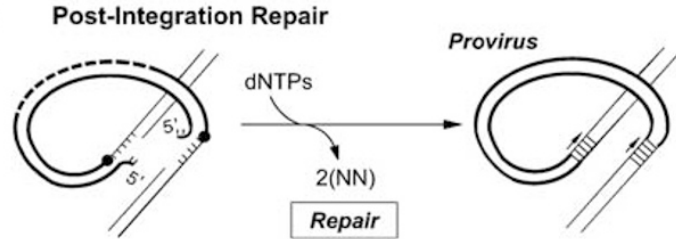

Figure 1 Stable, heritable establishment of a retroviral provirus requires (a) integrase-mediated DNA integration and (b) postintegration repair of the integration intermediate by host proteins. The shaded dumbbell-labeled (IN)n represents a multimer of integrase. $\mathrm{Me}^{2+}$ represents the required divalent metal cofactor, $\mathrm{Mg}^{2+}$ or $\mathrm{Mn}^{2+}$, and $\mathrm{N}$ represents any of the four bases. The dashed line in viral DNA signifies possible internal $(+)$ strand interruptions

\section{a Interruption of Chromatin Structure}
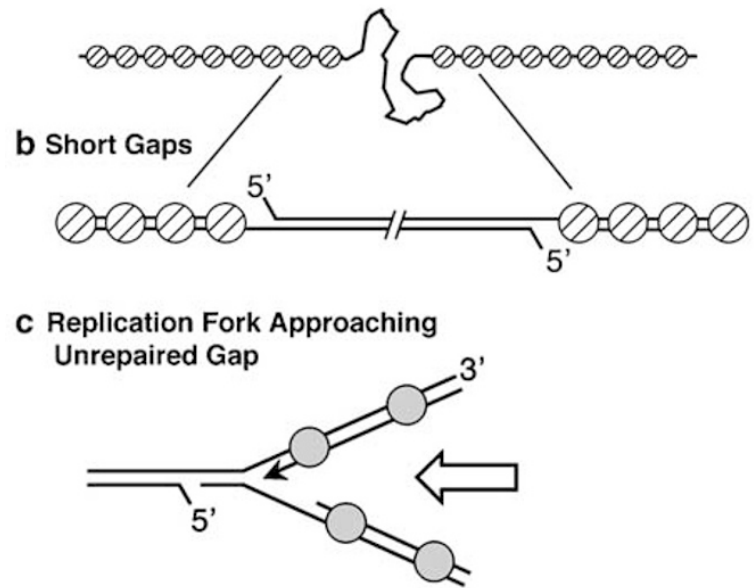

Figure 2 Retroviral DNA integration produces a complex lesion in host cell chromatin (a). Unrepaired DNA gaps that flank the integrated viral DNA (b) can become DSBs when encountered by a replication fork (c)

heritable association of retroviral DNA with host chromosomes.

\section{DNA Damage Response and Pathways for DSB Repair}

The genomes of all living cells are subject to damage that can occur spontaneously, or may be induced by various environmental agents, including viruses. Most types of damage are repaired by lesion-specific pathways, but some can escape correction and may cause stalling and/or collapse of replication forks during S-phase. Such events can lead either directly or indirectly to formation of DSBs, which can promote chromosomal rearrangement and deletions. Cells must encode robust systems to sense and repair such damage.
Cellular mechanisms that recognize DNA damage are very sensitive. For example, experiments with yeast cells have shown that even a single DSB can elicit a damage response and, if unrepaired, can lead to cell death. ${ }^{7-9}$ Such sensitivity implies a powerful surveillance and amplification system in which the highly conserved $\mathrm{PI}-3 \mathrm{~K}$-related protein kinases, Atm (ataxia-telangiectasia mutated) and Atr (Atm and Radrelated), play a pivotal role (Figure 3$).{ }^{10-12}$ These two kinases appear to posses both specialized and overlapping functions; although both respond to damage incurred by ionizing radiation (IR), Atm is especially critical for repair of DSBs, whereas Atr function appears to be most important following UV damage or replication fork stalling. ${ }^{10,11,13}$ Atr kinase is active in a complex with its regulatory partner Atrip, and appears to be specific for lesions that contain single strands that are coated with replication protein A (RPA).${ }^{14}$ Exactly how these protein kinases are activated by DNA damage is currently a subject of intense investigation. It is clear, however, that phosphorylation of target 'effector' proteins by Atm and Atr is required both to elicit cell-cycle checkpoint responses, which allow time for repair, and to recruit and/or activate repair proteins. In the absence of repair, signals transduced by these kinases can also trigger apoptosis.

The two major repair pathways for DSB repair in mammalian cells are described in Figure 4. In nonhomologous end joining (NHEJ), broken DNA ends are joined with no

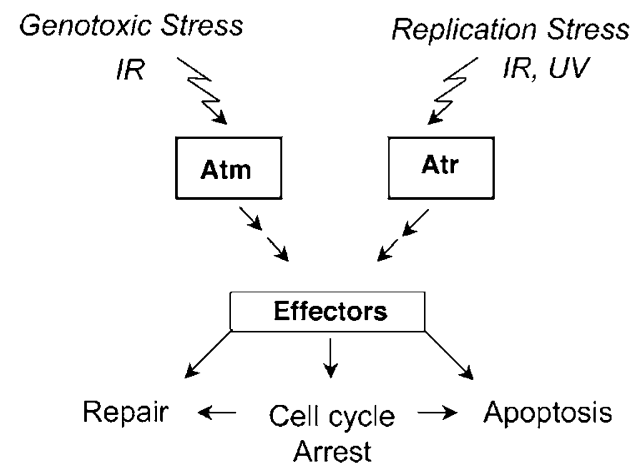

Figure 3 Atm and Atr are master regulators of the cell's DNA damage response

Major Pathways for DSB Repair in Mammalian Cells
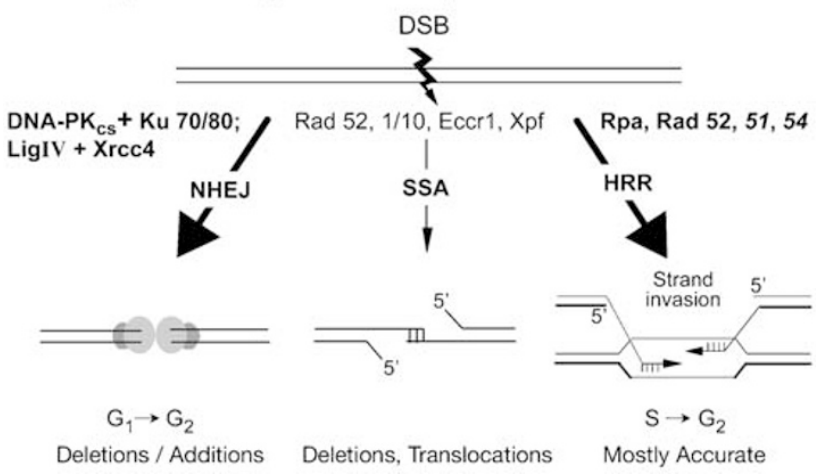

Figure 4 Two major pathways for DSB repair in mammalian cells are NHEJ and HRR 
requirement for homology. Heterodimers of $\mathrm{Ku} \mathrm{70/80} \mathrm{bind} \mathrm{to}$ these lesions and recruit a catalytic subunit (DNA-PKcs) to form an active DNA-dependent PI-3K-related protein kinase, known as DNA-dependent protein kinase (DNA-PK). The DNA ends are most likely held together in this complex where they may undergo some degree of processing to render them compatible for ligation by a ligase IV-Xrcc4 complex. Several proteins are phosphorylated during the process of repair and this modification is required for their activation. NHEJ can exact a genetic toll, as sequences may be lost or rearranged during the processing. This repair pathway has been studied most extensively in the context of immunoglobulin gene rearrangement $(\mathrm{V}(\mathrm{D}) \mathrm{J}$ recombination), which was first shown to depend on the activity of DNA-PKcs. NHEJ can take place from G1- through G2-phases of the cell cycle, but its relative contribution to DNA repair appears to be greatest in $\mathrm{G} 1$, when homologous recombination repair (HRR) is least prominent. ${ }^{15}$

In homologous recombination, broken ends are repaired by invading and copying sequences from an intact allele. In this repair pathway, the template preference appears to be heavily biased, with the sister chromatid preferred by two to three orders of magnitude. ${ }^{16}$ This implies that HRR occurs most frequently in newly replicated DNA ${ }^{17}$ and, not surprisingly, makes its greatest contribution during S- and G2-phases of the cell cycle. Repair promoted by HRR restores genetic content with high fidelity. However, in some (rare) cases, recombination between chromosomes can lead to loss of heterozygosity for distal markers, an event that can contribute to common human 'spontaneous' cancers. ${ }^{18}$ In a related but distinct pathway called single-strand annealing, the $5^{\prime}$-ends of broken DNA are resected, leaving $3^{\prime}$ single-end extensions. These single-stranded regions can then hybridize at short stretches of homology, for example, in directly repeated $\mathrm{Alu}$ sequences. Unlike HRR, this pathway creates deletions and also possibly translocations. Proteins specific to each of the major repair pathways have been identified in studies with yeast and human cells. ${ }^{19}$

\section{Evidence that Retroviral Postintegration Repair Depends on Host Cell Functions}

\section{Cell killing by retroviruses}

A potential role for the NHEJ-mediated DSB repair pathway in postintegration repair was first suggested by the work of Daniel et al. ${ }^{20}$ These studies showed that retroviral infection induces apoptosis in $80-90 \%$ of NHEJ-deficient cells (murine severe combined immunodeficient (scid) pre-B cells), with kinetics that were consistent with integration-induced damage. The response was independent of the expression of any gene products, as the vectors employed in these studies were either conditionally defective in viral gene expression (i.e. an avian sarcoma virus (ASV) vector) or contained no viral coding sequences (i.e. a human immunodeficiency virus type 1 (HIV-1) vector). However, these and subsequent studies $^{21,22}$ showed that the death of NHEJ-deficient cells was, in fact, dependent on the presence of active integrase in the infecting virion. Based on these results, it was proposed that retroviral DNA integration is perceived by cells as DNA damage, and that failure in postintegration repair triggers

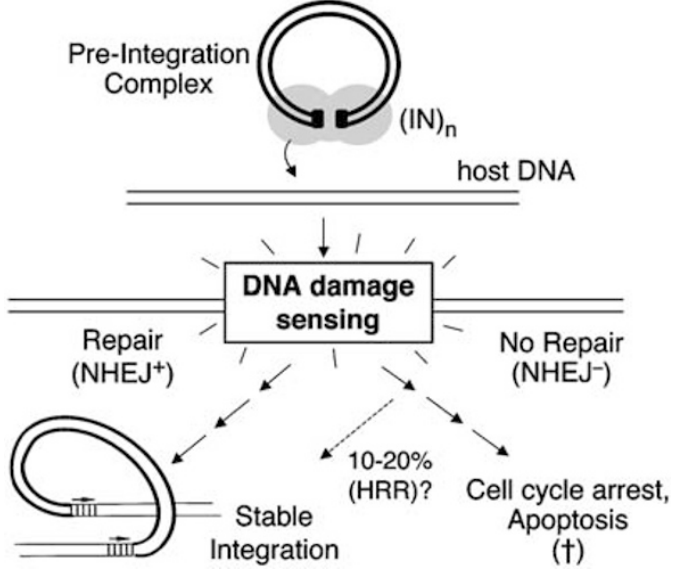

Figure 5 Model for the role of DNA damage sensing, NHEJ, and an alternative pathway in postintegration repair

apoptosis in NHEJ-deficient cells (Figure 5). It was also reported that Ty1 retrotransposition in yeast cells is dependent on the yeast $\mathrm{Ku}$ protein, ${ }^{23}$ suggesting a similar requirement in this system.

Subsequent studies that made use of the irreversible kinase inhibitor, wortmannin, ${ }^{21}$ showed that treatment with a concentration of the drug predicted to inhibit DNA-PKcs and Atm (but not Atr) sensitized normal cells to killing by integrasecompetent, but not integrase-defective retroviral vectors. However, because killing of cells deficient in DNA-PKcs was further potentiated by wortmannin (or Atm antisense DNA treatment), it was proposed that a second wortmanninsensitive reaction, catalyzed by Atm-dependent repair pathway, could provide a partial backup in the absence of NHEJ. As high fidelity postintegration repair occurs in the fraction of NHEJ-defective cells that survive retroviral infection, ${ }^{24}$ this backup pathway for postintegration repair must be quite accurate.

Results from a computer modeling approach indicated that a single hit (integration event) is sufficient to kill susceptible NHEJ-deficient cells. ${ }^{25}$ In these simulations, which included variables related to cell cycle, six mathematical models of integration-mediated killing were considered. The closest fit with experimental data was achieved by a model in which these NHEJ-deficient cell must pass through S-phase to activate apoptosis. Based on these studies, it was hypothesized that the interaction of a replication fork with an unrepaired integration intermediate during S-phase may be the trigger for the apoptotic response in NHEJ-deficient cells, due to the formation of DSBs. This model would be consistent with the observation that a single DSB is sufficient to kill a cell that is deficient in its repair. ${ }^{7-9}$

\section{Reduced retroviral transduction}

The ability of a retroviral vector to transduce a reporter gene depends on stable integration of the vector DNA. Consequently, assays of transduction efficiency provide a useful and convenient indicator for successful completion of postintegration repair and survival of the infected cells. Using this 
approach, it was shown that the efficiency of retroviral transduction of human and rodent cell that are deficient in NHEJ is only $10-20 \%$ of that observed with controls. ${ }^{20-22}$ Given the cell killing described above, reduced transduction efficiency is most readily explained as a result of apoptosis following abortive integration in these NHEJ repair-deficient cells. Consistent with this interpretation, wortmannin was found to reduce the efficiency of retroviral transduction of HeLa cells infected with ASV or HIV-1 vectors, with dose dependence that correlated with reduction of DNA-dependent protein kinase activity in these cells. Furthermore, although no reduction in transduction compared to controls was observed with Atm-deficient human (A-T) cells in the absence of the drug, transduction was virtually abolished in the presence of even the lowest concentration of wortmannin tested. ${ }^{21}$ The hypersensitivity of A-T cells to wortmannin may therefore be explained by the inhibition of DNA-PKcs in cells that posses no backup, Atm-dependent repair pathway. These results, together with the cell killing described above, support the idea that although Atm function is not normally required for postintegration repair, it contributes to the residual 10-20\% transduction observed in NHEJ-deficient cells (Figure 5).

\section{The role of NHEJ}

The first report of a role for NHEJ components in postintegration repair $^{20}$ was met with great interest, but also some skepticism, ${ }^{26}$ mainly because previous attempts to produce retrovirally transformed cell lines from scid mice did not reveal any obvious defect compared to normal mice. Indeed, the very scid lines used in the original studies of Daniel et al. ${ }^{20}$ had been derived by infecting bone marrow cells from scid mice with a retrovirus (Abelson murine leukemia virus). ${ }^{27}$ Although difficult to test, the failure to observe a defect in production of Abelson-transformed pre-B cells may be explained by the survival of a fraction of the infected scid cells, due to repair via the backup pathway, and strong selection for the infected survivors whose proliferation would be enhanced by expression of the v-abl oncogene. Subsequent studies from several laboratories have shown that deficiencies in NHEJ result in reduced retrotransposition, retroviral DNA integration and transduction, or subsequent cell survival, consistent with a critical interplay between retroviruses/retrotransposons and components of this repair pathway. ${ }^{5,6,23,28,29}$ However, two publications have challenged a role for NHEJ in postintegration repair because of apparent discrepancies in the timing of retrovirus-mediated killing of NHEJ-deficient cells and the effect of an integration-defective vector. These findings were interpreted to suggest that the NHEJ pathway is not required for postintegration repair following lentiviral (i.e. HIV) infection, ${ }^{28}$ or is acting solely on unintegrated viral DNA. ${ }^{5}$ More recent, independent experiments ${ }^{22}$ failed to reproduce the discrepancies in one earlier report $^{28}$ and showed that experimental conditions likely to produce a very high multiplicity of infection (m.o.i.) could explain the discrepancies in the second report. ${ }^{5}$ To date, the bulk of available evidence supports a role for NHEJ in postintegration repair for both HIV and the simpler retroviruses, such as ASV.

\section{Evidence that Integration is Perceived as DNA Damage by the Cell}

\author{
Histone H2AX is phosphorylated at sites of \\ retroviral DNA integration
}

The evolutionarily conserved histone $\mathrm{H} 2 \mathrm{AX}$ comprises from about 2 to $25 \%$ of the histone $\mathrm{H} 2 \mathrm{~A}$ pool in mammalian cells and is distributed randomly in nucleosomes. ${ }^{30}$ The extended C-terminal tail of H2AX contains an SQE motif that is a target for serine phosphorylation by DNA-PK, Atm, and Atr. ${ }^{31}$ This $\mathrm{H} 2 \mathrm{AX}$ serine residue is rapidly phosphorylated in large chromatin domains flanking sites of DSBs produced by genotoxic treatments and stalled replication forks. ${ }^{32-34}$ Phosphorylated histone $\mathrm{H} 2 \mathrm{AX}$, denoted $\gamma-\mathrm{H} 2 \mathrm{AX}$, can be detected as microscopically visible foci at such sites upon staining with a specific antibody. This histone modification seems to play an important role in the processing or repair of such lesions. ${ }^{35,36} \gamma-\mathrm{H} 2 \mathrm{AX}$ has also been observed at sites of $\mathrm{V}(\mathrm{D}) \mathrm{J}$ recombination, ${ }^{37}$ meiotic strand breaks, ${ }^{38}$ and other physiological programmed reactions in which DSBs are formed. ${ }^{39}$

It was recently discovered that retroviral DNA integration also promotes formation of $\gamma-\mathrm{H} 2 \mathrm{AX}$, as detected by foci formation and chromatin immunoprecipitation methods. ${ }^{40}$ This comprises the first direct evidence for association of newly integrated viral DNA with a protein species that is an established marker for the onset of a DNA damage response. On the other hand, transduction experiments with $\mathrm{H} 2 \mathrm{AX}-1-$ knockout and control cells indicated that, as with $V(D) J$ recombination, this histone is dispensable for postintegration repair. These observations lend support to a model in which $\gamma$ H2AX promotes DNA repair of DSBs by anchoring broken ends. ${ }^{41-44}$ As chromosomal breaks are likely to be held together by the RAG1/2 complex during $V(D) J$ recombination, ${ }^{45}$ and are linked covalently by viral DNA in retroviral DNA integration (Figure 1), a $\gamma-\mathrm{H} 2 \mathrm{AX}$-anchoring function is predicted to be dispensable in both reactions.

\section{Atr plays a critical role in postintegration repair}

Pharmacological studies using the Atm and Atr inhibitor caffeine, and cells that express conditionally a dominantnegative, kinase-dead Atr protein (Atr-kd) have provided evidence that retroviral DNA integration elicits an Atrdependent DNA damage response. ${ }^{46}$ The transduction efficiency of two retroviral vectors (HIV-1 and ASV) was found to be decreased in the presence of concentrations of caffeine that had little or no effect on early steps in infection that precede integration. The amount of viral DNA physically joined to host DNA was also reduced in the presence of caffeine. Analyses with cells that overexpressed Atr-kd suggested that these reductions could be explained by the death of cells that were unable to repair the damage caused by viral DNA integration. What aspect of postintegration repair might depend on ATR? As noted earlier, Atr is a major sensor of DNA damage, ${ }^{10,11}$ and appears to be specific for RPAcoated single-stranded DNA. ${ }^{14}$ Its activity is required in cellular responses to ionizing and UV irradiation as well as collapsed replication forks. ${ }^{10,11,47}$ It is possible that 
RPA-coated single-stranded regions in integration intermediates recruit Atr as they are formed. One might also speculate that the damage caused when a replication fork encounters an unrepaired integration intermediate may be similar to other replication fork catastrophes in which an Atr has been proposed to play a pivotal role. ${ }^{14,47}$ As illustrated in Figure 3, signaling through Atr-mediated phosphorylation of various effector molecules might lead to transient cell-cycle arrest, as well as the recruitment or activation of proteins necessary for repair. Failure in either or both processes could render an unrepaired integration intermediate unstable and lead to cell death.

The majority of evidence to date indicates that cells respond to damage incurred during retroviral integration just as they do to DSBs induced by treatment with IR or certain genotoxic drugs. These findings contradict the generally held notion that production of free DNA ends is the signal for initiating this damage/repair response. As illustrated in Figures 1 and 2, free double-strand ends are not produced during retroviral DNA integration. How might integration and IR elicit the same response? Three hypotheses may be considered to explain this result: (i) Transmission of the damage signal may not depend on recognition of free DNA ends but rather on some structural or conformational change in chromatin. This idea has received support recently from studies of Atm activation ${ }^{12}$ and Atr-dependent activities. ${ }^{32}$ (ii) The integration intermediate resembles DNA structures produced during repair of free DNA generated by IR and is thereby recognized and acted upon by the NHEJ pathway (Figure 6). ${ }^{48}$ Hypotheses (i) and (ii) are not mutually exclusive. Finally, (iii) free DNA ends may be the trigger for the damage response during retroviral DNA integration in cycling cells as follows: although genetic studies have shown that integration can occur both before and after passage of a replication fork in S-phase, ${ }^{49}$ it is conceivable that postintegration repair reactions are activated primarily via a damage response to a DSB that forms when a replication fork encounters the flanking gap during S-phase (Figure 2c), and is then repaired by NHEJ or, in some cases, by the Atmdependent backup pathway. We note, however, that the DNA of some retroviruses, such as HIV and ASV, can be integrated stably in noncycling cells. ${ }^{50-55}$ In such cases, activation of repair pathways cannot depend on passage through S-phase.

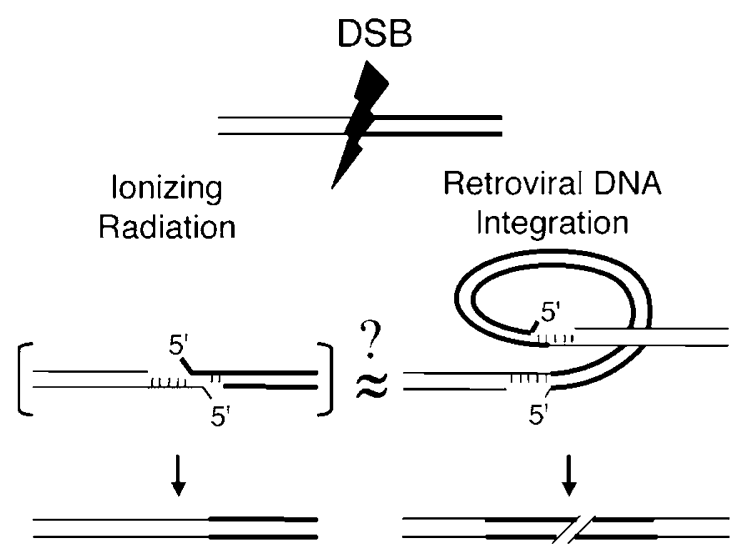

Figure 6 Similar structural features in intermediates of NHEJ repair of DSB produced by $I R$ and the retroviral DNA integration intermediates

\section{Cytopathic Effects of Retroviral Infection and Possible Relevance to DNA Damage Signaling Pathways}

Studies of the cytopathic effects that are characteristic of certain retroviral strains has led to the hypothesis that viral components or replication intermediates may activate the host DNA damage-sensing/repair systems during infection. ${ }^{56-61}$ Two different mechanisms have been considered.

Several lines of research, beginning with the early studies of Temin and colleagues, ${ }^{60}$ have led to the idea that unintegrated viral DNA may induce cell killing. ${ }^{58,59,62}$ It was recently suggested that high concentrations of unintegrated viral DNA, containing free ends, might be sensed as irreparable DNA damage in the cell, and that the NHEJ system may suppress an apoptotic response by joining such ends to form circles, which are a dead-end product. ${ }^{5,63}$ However, other experiments have shown that the accumulation of unintegrated viral DNA and the reduced formation of circles can be uncoupled from cell killing. ${ }^{29,59,62}$ It should also be noted that high concentrations of unintegrated DNA would normally be associated with high frequency of integration, which may be toxic to cells. The effects of high DNA concentration and highfrequency integration on cell survival can be tested by manipulating experimental conditions, that is, by using a high m.o.i., or infection methods that increase the effective m.o.i. Under these conditions, a specific NHEJ deficiency (Lig 4-/-) results in increased cytopathic effects as compared to control cells, but there is some controversy as to whether this is due to unintegrated DNA or unrepaired integration intermediates. ${ }^{5,22,63}$ It is, perhaps, not surprising that the high concentrations of viral DNA generated by experimental manipulations might produce toxic effects in wild-type or NHEJ-deficient cells; however, the relevance of these effects to natural infections remains unclear.

A second mechanism, mediated by $\mathrm{Vpr}$, has been proposed to explain how the DNA damage response pathway may be involved in the cytopathology of HIV-1. Vpr is a small, multifunctional, auxiliary protein that is assembled into the HIV-1 virion. Early after infection, the virion-associated Vpr is able to mediate transient G2 cell-cycle arrest, ${ }^{64}$ a condition that may favor efficient HIV-1 transcription. ${ }^{57}$ Other experiments have shown that when expressed independently from a vector, Vpr can induce apoptosis. It was also shown that such expression can activate Atr and cause formation of $\gamma-\mathrm{H} 2 \mathrm{AX}$ foci. ${ }^{61}$ It should be noted that other investigators have observed that such independently expressed Vpr elicits a wide range of abnormalities in mitosis, cytokinesis, and nuclear structure. ${ }^{56}$ It seems possible, therefore, that independent expression or overexpression of Vpr might produce cellular stresses that activate Atr indirectly. As mentioned in the previous section, chromosomal DNA damage produced by ASV DNA integration induces $\gamma-\mathrm{H} 2 \mathrm{AX}$ focus formation, ${ }^{40}$ and a recent report has presented evidence that Atr is required for efficient transduction by HIV vectors that lack Vpr. ${ }^{65}$ It will be important, therefore, to develop methods that can distinguish between the DNA damage response that might be attributed to virion-associated Vpr following infection with HIV and the effects related to postintegration repair. 
In summary, DNA damage responses have been associated with the intracellular accumulation of large amounts of retroviral DNA and with independent expression of HIV-1 Vpr. In both cases, the concentrations or expression of these viral components have been manipulated experimentally. It is possible that similar conditions could exist in an environment in which there is a very high density of both susceptible cells and virus, for example, in the germinal centers of lymph nodes during HIV-1 infection. However, at present, the relevance of these observations to viral cytopathology remains uncertain.

\section{Implications}

This review has focused mainly on the cellular response to retroviral infection in NHEJ-deficient cycling cells. Infection of NHEJ-deficient, lymphocyte-derived cultures cells has been shown to provoke cell killing. ${ }^{20,22,28,29}$ Animal cells differ in their propensities to respond to DNA damage by apoptosis. Lymphocytes appear to be primed for such a response, perhaps because it is required during normal immune cell selection. A single unrepaired integration event can trigger apoptosis in such cells. ${ }^{25}$ However, integration can also lead to apoptotic cell death in Atr-deficient human fibroblastic cells. ${ }^{46}$ Reduced transduction efficiencies of these, and other cells that have genetic or drug-induced deficiencies in NHEJ or Atr function, are most likely attributable to integrationinduced cell killing. Both p53-dependent and -independent pathways have been described for DNA damage-induced checkpoint activation and apoptosis (reviewed in Durocher and Jackson, ${ }^{10}$ Shiloh, ${ }^{11}$ and Norbury and Zhivotovsky ${ }^{66}$ ). As reduced transduction is observed in wortmannin-treated HeLa cells, ${ }^{21}$ which are p53 deficient, this tumor suppressor protein is not likely to be essential for integration-induced killing. The roles of other downstream effectors of this process remain to be elucidated.

One obvious implication of these findings is that during normal infection in repair-competent cells, recruitment of DNA damage-signaling and repair proteins to the integration site is essential for survival of both the host cell and the virus. ${ }^{22,46}$ As the establishment of a stably integrated provirus generally does not cause deleterious effects in the host cell, it can be assumed that in repair-proficient cells (i) damage to the host chromosome produced by integration is, indeed, repaired and (ii) unintegrated linear viral DNA is somehow protected, such that it is not recognized as a damaged chromosomal fragment that needs to be 'repaired', before it is integrated. Additional experiments will be required to determine exactly how and when cellular damage response and repair pathway components participate in postintegration repair. Such details may suggest new approaches and strategies for development of antiviral therapies, based on cellular targets.

Targeting cellular proteins and pathways for antiretroviral therapy would be especially attractive in light of the high mutation rate of retroviruses, and the rapid selection for mutants that can escape inhibitors of retrovirally encoded proteins. Some cellular proteins that are already implicated in postintegration repair, in particular those not required for cell viability, might prove to be useful as targets for inhibition in some circumstances. However, it is possible that proteins that function at other steps in the pathways that are critical for postintegration repair may be better suited as drug targets. In this context, it will be important to determine if any pathway component interacts directly or specifically with retroviral proteins, as such interactions might provide unique opportunities for inhibition. Some practical applications of the available knowledge may be possible, even in the short term. For example, rescue of NHEJ-deficient cells from HIV killing could provide a valuable, cell-based assay for small molecule inhibitors of any early step in retroviral infection, up to and including postintegration repair. ${ }^{21}$

Retroviral systems should also be useful to investigate how the cellular pathways that govern chromatin repair, cell-cycle arrest, and apoptosis are coordinated. Elucidation of mechanisms by which viruses exploit or circumvent host functions has contributed enormously to our understanding of cell molecular biology in the past. Although much has been learned through the study of damage induced by genotoxic treatments, it is often difficult to draw inferences about molecular mechanisms related to the response of such lesions, because the damage to cellular DNA is extensive and/or heterogeneous. These general limitations do not apply to the retroviral system because the nature of the lesion is known and the number of lesions or 'hits' can be predetermined by adjusting virus titer. Furthermore, as already demonstrated, ${ }^{40}$ retroviral DNA (3$10 \mathrm{~kb}$ ) can serve as a valuable marker of the damage site in whole cells or subcellular fractions, and the timing of the integration event can also be monitored. Further study of the molecular mechanisms that mediate postintegration repair should provide new insights into the cellular pathways that are required to maintain genetic stability or, in its absence, to program cell death.

\section{Acknowledgements}

This work was supported by National Institutes of Health grants Al40385, CA71515, CA06927, and also by an appropriation from the Commonwealth of Pennsylvania. The contents of this manuscript are solely the responsibility of the authors and do not necessarily represent the official views of the National Cancer Institute, or any other sponsoring organization.

\section{References}

1. Coffin JM, Hughes SH and Varmus HE (1997) Retroviruses. Plainview, NY: Cold Spring Harbor Laboratory Press pp. 161, Chapter 5

2. Flint SJ, Enquist LW, Krug RM, Racaniello VR and Skalka AM (2000) Principles of Virology. Molecular Biology, Pathogenesis, and Control. Washington, DC: ASM Press pp. 230 Chapter 7

3. Miller MD, Wang B and Bushman FD (1995) Human immunodeficiency virus type 1 preintegration complexes containing discontinuous plus strands are competent to integrate in vitro. J. Virol. 69: 3938-3944

4. Lee YMH and Coffin JM (1991) Relationship of avian retrovirus DNA synthesis to integration in vitro. Mol. Cell. Biol. 11: 1419-1430

5. Li L, Olvera JM, Yoder KE, Mitchell RS, Butler SL, Lieber M, Martin SL and Bushman FD (2001) Role of the non-homologous DNA end joining pathway in the early steps of retroviral infection. EMBO J. 20: 3272-3281

6. Jeanson L, Subra F, Vaganay S, Hervy M, Marangoni E, Bourhis J and Mouscadet J-F (2002) Effect of Ku80 depletion on the preintegrative steps of HIV-1 replication in human cells. Virology 300: 100-108 
7. Olive PL (1998) The role of DNA single- and double-strand breaks in cell killing by ionizing radiation. Radiat. Res. 150: S42-S51

8. Ho KS and Mortimer RK (1973) Induction of dominant lethality by X-rays in radiosensitive strain of yeast. Mutat. Res. 20: 45-51

9. Bennett CB, Lewis AL, Baldwin KK and Resnick MA (1993) Lethality induced by a single site-specific double-strand break in a dispensable yeast plasmid. Proc. Natl. Acad. Sci. USA 90: 5613-5617

10. Durocher D and Jackson SP (2001) DNA-PK, ATM and ATR as sensors of DNA damage: variations on a theme? Curr. Opin. Genet. Dev. 13: 225-231

11. Shiloh Y (2001) ATM and ATR: networking cellular responses to DNA damage. Curr. Opin. Genet. Dev. 11: 71-77

12. Bakkenist CJ and Kastan MB (2003) DNA damage activates ATM through intermolecular autophosphorylation and dimer dissociation. Nature 421: 499-506

13. Abraham RT (2001) Cell cycle checkpoint signaling through the ATM and ATR kinases. Genes Dev, 15: 2177-2196

14. Zou L and Elledge SJ (2003) Sensing DNA damage through Atrip recognition of RPA-ssDNA complexes. Science 300: 1542-1548

15. Rothkamm K, Krüger I, Thompson LH and Löbrich M (2003) Pathways of DNA double-strand break repair during the mammalian cell cycle. Mol. Cell. Biol. 23 5706-5715

16. Johnson RD and Jasin M (2001) Double-strand-break-induced homologous recombination in mammalian cells. Biochem. Soc. Trans. 29: 196-201

17. Courcelle J, Donaldson JR, K-H C and Courcelle CT (2003) DNA damageinduced replication fork regression and processing in Escherichia coli. Science 299: 1064-1067

18. Vilenchik MM and Knudson AG (2003) Endogenous DNA double-strand breaks: production, fidelity of repair, and induction of cancer. Proc. Natl. Acad. Sci. USA 100: 12871-12876

19. Valerie K and Povirk LF (2003) Regulation and mechanisms of mammalian double-strand break repair. Oncogene 22: 5792-5812

20. Daniel R, Katz RA and Skalka AM (1999) A role for DNA-PK in retroviral DNA integration. Science 284: 644-647

21. Daniel R, Katz RA, Merkel G, Hittle JC, Yen TJ and Skalka AM (2001a) Wortmannin potentiates integrase-mediated killing of lymphocytes and reduces the efficiency of stable transduction by retroviruses. Mol. Cell. Biol. 21: 1164-1172

22. Daniel R, Greger JG, Katz RA, Taganov KD, Wu X, Kappes JC and Skalka AM (2004) Evidence that stable retroviral transduction and cell survival following DNA integration depend on components of the nonhomologous end joining repair pathway. J. Virol. 78: 8573-8581

23. Downs JA and Jackson SP (1999) Involvement of DNA end-binding protein Ku in Ty element retrotransposition. Mol. Cell. Biol. 19: 6260-6268

24. Taganov K, Daniel R, Katz RA, Favorova $O$ and Skalka AM (2001) Characterization of retrovirus-host DNA junctions in cells deficient in nonhomologous-end joining. J. Virol. 75: 9549-9552

25. Daniel R, Litwin S, Katz RA and Skalka AM (2001b) Computational analysis of retrovirus-induced scid cell death. J. Virol. 75: 3121-3128

26. Coffin JM and Rosenberg N (1999) Closing the joint. Nature 399: 413-416

27. Schuler W, Weiler IJ, Schuler A, Phillips RA, Rosenberg N, Mak TW, Kearney JF, Perry RP and Bosma MJ (1986) Rearrangement of antigen receptor genes is defective in mice with severe combined immune deficiency. Cell 46 : 963-972

28. Baekelandt V, Claeys A, Cherepanov P, De Clercq E, De Strooper B, Nuttin B and Debyser Z (2000) DNA-dependent protein kinase is not required for efficient lentivirus integration. J. Virol. 74: 11278-11285

29. Lau A, Kanaar R, Jackson SP and O'Connor MJ (2004) Suppression of retroviral infection by the RAD52 DNA repair protein. EMBO J. 23: 3421-3429 (see especially Supplementary Data)

30. Rogakou EP, Pilch DR, Orr AH, Ivanova VS and Bonner WM (1998) DNA double-stranded breaks induce histone H2AX phosphorylation on serine 139 J. Biol. Chem. 273: 5858-5868

31. Celeste A, Fernandez-Capetillo O, Kruhlak MJ, Pilch DR, Staudt DW, Lee A Bonner RF, Bonner WM and Nuzzenzweig A (2003) Histone H2AX phosphorylation is dispensable for the initial recognition of DNA breaks. Nat. Cell Biol. 5: 675-679

32. Ward IM and Chen J (2001) Histone H2AX is phosphorylated in an ATR dependent manner in response to replicational stress. J. Biol. Chem. 276 47759-47762
33. Rogakou EP, Boon C, Redon C and Bonner WM (1999) Megabase chromatin domains involved in DNA double-strand breaks in vivo. J. Cell Biol. 146: 905-916

34. Redon C, Pilch DR, Rogakou EP, Sedelnikova OA, Newrock K and Bonner WM (2002) Histone H2A variants H2AX and H2AZ. Curr. Opin. Genet. Dev. 12: 162-169

35. Celeste A, Petersen S, Romanienko PJ, Fernandez-Capetillo O, Chen H-T, Sedelnikova OA, Reina-San-Martin B, Coppola V, Meffre E, Difilippantonio MJ, Redon C, Pilch DR, Olaru A, Eckhaus M, Camerini-Otero DC, Tessarollo L, Livak F, Manova K, Bonner WM, Nussenzweig MC and Nussenzweig A (2002) Genomic instability in mice lacking histone H2AX. Science 296: 922-927

36. Bassing $\mathrm{CH}$, Chua KF, Sekiguchi J, Suh H, Whitlow SR, Fleming JC, Monroe BC, Ciccone DN, Yan C, Vlasakova K, Livingston DM, Ferguson DO, Scully R and Alt FW (2002) Increased ionizing radiation sensitivity and genomic instability in the absence of histone H2AX. Proc. Natl. Acad. Sci. USA 99: 8173-8178

37. Chen HT, Bhandoola A, Difilippantonia MJ, Zhu J, Brown MJ, Tai X, Rogakou EP, Brotz TM, Bonner WM, Ried T and Nussenzweig A (2000) Response to RAG-mediated VDJ cleavage by NBS1 and gamma-H2AX. Science 290: 1962-1965

38. Fernandez-Capetillo O, Mahadevaiah SK, Celeste A, Romanienko PJ, Camerini-Otero RD, Bonner WM, Manova K, Burgoyne P and Nussenzweig A (2003a) H2AX is required for chromatin remodeling and inactivation of sex chromosomes in male mouse meiosis. Dev. Cell 4: 497-508

39. Petersen S, Casellas R, Reina-San-Martin B, Chen HT, Difilippantonio MJ, Wilson PC, Hanitsch L, Celeste A, Muramatsu M, Pilch DR, Redon C, Ried T, Bonner WM, Honjo T, Nussenzweig MC and Nussenzweig A (2001) AID is required to initiate NBs $1 / \mathrm{g}-\mathrm{H} 2 \mathrm{AX}$ focus formation and mutations at sites of class switching. Nature 414: 660-665

40. Daniel R, Ramcharan J, Rogakou EP, Taganov KD, Greger JG, Bonner WM, Nussenzweig A, Katz RA and Skalka AM (2004) Histone H2AX is phosphorylated at sites of retroviral DNA integration, but is dispensable for post-integration repair. J. Biol. Chem. 279: 45810-45814

41. Celeste A, Difilippantonio S, Difilippantonio MJ, Fernandez-Capetillo O, Pilch DR, Sedelnikova OA, Eckhaus M, Ried T, Bonner WM and Nussenzweig A (2003) H2AX haploinsufficiency modifies genomic stability and tumor susceptibility. Cell 114: 371-383

42. Bassing $\mathrm{CH}$, Suh $\mathrm{H}$, Ferguson DO, Chua KF, Manis J, Eckersdorff $M$, Gleason M, Bronson R, Lee C and Alt FW (2003) Histone H2AX: a dosage-dependent suppressor of oncogenic translocations and tumors. Cell 114: 359-370

43. Bassing $\mathrm{CH}$ and Alt FW (2004) $\mathrm{H} 2 \mathrm{AX}$ may function as an anchor to hold broken chromosomal DNA ends in close proximity. Cell Cycle 3: 149-153

44. Fernandez-Capetillo O, Lee A, Nussenzweig MC and Nussenzweig A (2004) H2AX: the histone guardian of the genome. DNA Repair 3: 959-967

45. Jones JM and Gellert M (2003) Autoubiquitylation of the V(D)J recombinase protein RAG1. Proc. Natl. Acad. Sci. USA 100: 15446-15451

46. Daniel R, Kao G, Taganov K, Greger J, Favorova O, Merkel G, Yen TJ, Katz RA and Skalka AM (2003) Evidence that the DNA integration process triggers an ATR-dependent DNA damage response. Proc. Natl. Acad. Sci. USA 100: 4778-4783

47. Tibbetts RS, Cortez D, Brumbaugh KM, Scully R, Livingston DM, Elledge SJ and Abraham RT (2000) Functional interactions between BRCA1 and the checkpoint kinase ATR during genotoxic stress. Genes Dev. 14: 2989-3002

48. Tseng H-M and Tomkinson AE (2002) A physical and functional interaction between yeast pol4 and Dn14-Lif1 links DNA synthesis and ligation in nonhomologous end joining. J. Biol. Chem. 47: 45630-45637

49. Katz RA, Greger JG, Boimel P and Skalka AM (2003) Human immunodeficiency virus type 1 DNA nuclear import and integration are mitosis independent in cycling cells. J. Virol. 77: 13412-13417

50. Bukrinsky MI, Sharova N, Dempsey MP, Stanwick TL, Bukrinskaya AG, Haggerty $S$ and Stevenson M (1992) Active nuclear import of human immunodeficiency virus type 1 preintegration complexes. Proc. Natl. Acad. Sci. USA 89: 6580-6584

51. Naldini L, Blömer U, Gallay P, Ory D, Mulligan R, Gage FH, Verma IM and Trono D (1996) In vivo gene delivery and stable transduction of nondividing cells by a lentiviral vector. Science 272: 263-267 
52. Weinberg JB, Matthews TJ, Cullen BR and Malim MH (1991) Productive human immunodeficiency virus type 1 (HIV-1) infection of non-proliferating human monocytes. J. Exp. Med. 174: 1477-1482

53. Hatziioannou T and Goff SP (2001) Infection of nondividing cells by Rous sarcoma virus. J. Virol. 75: 9526-9531

54. Katz RA, Greger JG, Darby K, Boimel P, Rall GF and Skalka AM (2002) Transduction of interphase cells by avian sarcoma virus. J. Virol. 76: 5422-5434

55. Greger JG, Katz RA, Taganov K, Rall GF and Skalka AM (2004) Transduction of terminally differentiated neurons by avian sarcoma virus. J. Virol. 78: 4902-4906

56. Chang F, Re F, Sebastian S, Sazer S and Luban J (2004) HIV-1 Vpr induces defects in mitosis, cytokinesis, nuclear structure and centrosomes. Mol. Cell. Biol. 15: 1793-1801

57. Gummuluri S and Emerman M (1999) Cell cycle- and Vpr-mediated regulation of human immunodeficiency virus type 1 expression in primary and transformed T-cell lines. J. Virol. 73: 5422-5430

58. Maury W, Wright PJ and Bradley S (2003) Characterization of a cytolytic strain of equine infectious anemia virus. J. Virol. 77: 2385-2399

59. Nanua S and Yoshimura FK (2004) Differential cell killing by lymphomagenic murine leukemia viruses occurs independently of p53 activation and mitochondrial damage. J. Virol. 78: 5088-5096
60. Weller SK, Joy AE and Temin HM (1980) Correlation between cell killing and massive second-round superinfection by members of some subgroups of avian leukosis virus. J. Virol. 33: 494-506

61. Zimmerman ES, Chen J, Andersen JL, Ardon O, Dehart JL, Blackett J, Choudhary SK, Camerini D, Nghiem P and Planelles V (2004) Immunodeficiency virus type $1 \mathrm{Vpr}$-mediated G2 arrest requires Rad17 and Hus1 and induces nuclear BRCA1 and gamma-H2AX focus formation. Mol. Cell. Biol. 24: 9286-9294

62. Bergeron L and Sodroski J (1992) Dissociation of unintegrated viral DNA accumulation from single-cell lysis induced by human immunodeficiency virus type 1. J. Virol. 66: 5777-5787

63. Kilzer JM, Stracker TH, Beitzel B, Meek K, Weitzman MD and Bushman FD (2003) Roles of host cell factors in circularization of retroviral DNA. Virology 314: 460-467

64. Poon B, Grovit-Ferbas K, Stewart SA and Chen IS (1998) Cell cycle arrest by $\mathrm{Vpr}$ in HIV-1 virions and insensitivity to antiretroviral agents. Science 281: 266-269

65. Daniel R, Marusich E, Argyris E, Zhao RY, Skalka AM and Pomerantz RJ (2004) Caffeine inhibits HIV-1 transduction of non-dividing cells. J. Virol. 79: 2058-2065

66. Norbury CJ and Zhivotovsky B (2004) DNA damage-induced apoptosis. Oncogene 23: 2797-2808 